CAHIERS DE

NARRATOLOGIE
Cahiers de Narratologie

Analyse et théorie narratives

$10.2 \mid 2001$

La voix narrative

\title{
La narrative médiévale ou la viabilité de l'impossible: La casa de Hércules
}

\section{Michel Sauve}

\section{(2) OpenEdition}

1 Journals

Édition électronique

URL : http://journals.openedition.org/narratologie/10289

DOI : 10.4000/narratologie.10289

ISSN : 1765-307X

Éditeur

LIRCES

\section{Édition imprimée}

Date de publication : 1 janvier 2001

Pagination : 425-439

ISBN : 2914561032

ISSN : 0993-8516

Référence électronique

Michel Sauve, "La narrative médiévale ou la viabilité de l'impossible : La casa de Hércules ", Cahiers de Narratologie [En ligne], 10.2 | 2001, mis en ligne le 01 janvier 2001, consulté le 11 juin 2020. URL

http://journals.openedition.org/narratologie/10289; DOI : https://doi.org/10.4000/narratologie.10289 


\title{
LA NARRATIVE MÉDIÉVALE OU LA VIABILITÉ DE L'IMPOSSIBLE : LA CASA DE HÉRCULES.
}

\author{
Michel SAUVE \\ Université du Mans
}

Curieux épisode que celui de la Chronique de $1344^{1}$ où le roi Rodrigue - au demeurant, est-il bien roi ? ${ }^{1 a}-$, à peine intronisé, n'a de cesse de découvrir le secret - à moins que ce ne soit un trésor - qui gît, maintes fois cadenassé, en les murs de la maison d'Hercule, à Tolède - au mépris des conseils que lui donnent de s'en abstenir les gardiens épouvantés de ce succédané espagnol du temple de Vesta.

Curieux à plus d'un titre: au plan fonctionnel, il dit, certes, l'inéluctable, mais son absence n'y retrancherait rien ; il paraît donc avoir un rôle esthétique plus que vraiment fondamental. Et pourtant, on sent bien que l'intention n'en est pas simplement ornementale : chargé du merveilleux des contes dont s'éclaire la grisaille des longs après-midi des

${ }^{1}$ Cet article représentant l'aboutissement d'un projet fait il y a une quinzaine d'années, à Lille, à l'occasion d'un simple cours d'agrégation, nous écarterons ici toute prétention à l'érudition bibliographique ou documentaire, et nous contenterons de nous appuyer, tant pour ce qui est de la Crónica de 1344 que pour ce qui se rapporte à la Crónica Sarracina, sur l'ouvrage alors proposé aux étudiants, la Floresta de leyendas heroicas españolas - Rodrigo, el último godo, T. I, compilación, prólogo y notas de Ramón Menéndez Pidal, Clásicos castellanos, ed. Espasa-Calpe, Madrid, 1973.

1a C'est en fait un tyran qu'on nous présente. «Fizo fazer a los de España que lo tomasen por rey ", nous dit-on: "il fit faire à ceux d'Espagne [telle erreur] qu'ils le prissent pour roi », ou, au moins, «il obtint de ceux d'Espagne qu'ils le prissent pour roi "; indubitablement, le texte ne lui est pas favorable, et rejoint le point de vue traditionnellement attribué aux partisans de Witiza : Rodrigue a, en tout cas, sollicité son élection ; ainsi, ne serait-ce que du fait de l'usurpation évoquée à demi-mots, l'accès à la maison sacrée aurait dû lui être interdit. 
enfants alités, il est aussi dramatique dans la mesure où, compliquant un peu l'action, il lui donne le poids, la résonance qu'à soi seul, le récit, que d'ailleurs il encadre et enserre, de la mésaventure d'Alataba, fija del conde don Illán, ne susciterait peut-être pas aussi pleinement ; il est, en outre, pathétique, puisqu'il permet en sa conclusion de rendre tangible le trouble de Rodrigue devant l'erreur consommée - thème bien mieux exploité, du reste, en des termes qu'on dirait imprégnés de vécu réel, et mis dans la bouche des chevaliers de la suite royale, par Pedro de Corral, dans sa Chronique Sarrazine.

Curieux aussi parce que, dans un texte à prétention historique, puisqu'il vise à conter de l'advenu, ou ce qui est tenu pour tel, il met en scène - tout à coup érigé en haut lieu d'une intégrité "nationale » dont la prise de conscience ne remonte pas à bien longtemps - un édifice surgi du néant, et qui n'a très probablement jamais eu d'existence autre qu'imaginaire.

Si l'on en croit R. Menéndez $\mathrm{Pidal}^{2}$, l'épisode - qui nous apparaît effectivement comme le négatif, fidèle à quelques détails près, de l'aventure d'Ali Baba - serait de tradition orientale : le thème de la maison fermée aurait été repris par les Arabes à partir de récits d'origine perse, et peut-être chinoise - ou indienne, suggère même A. H. Krappe ${ }^{2 a}$. Cela pourtant ne suffit pas pour éclairer l'implication, dans cette affaire, d'Hercule, héros en qui s'incarnait, pour l'antiquité grecque, la gloire d'Héra ${ }^{3}$, et que ses exploits ont rendu panméditerranéen bien avant qu'en l'an 622 du comput chrétien l'Islam ne vît même le jour : à ce point qu'il est tentant de voir en lui le symbole mythologique de la mainmise progressive des marins-commerçants hellènes sur le monde qui les entourait ${ }^{4}$.

Pourquoi donc, en cette relation de faits d'une époque si troublée et si sombre, cette référence au demi-dieu le plus

2 Op. cit.

2a In La légende de la Maison fermée de Tolède, Bulletin Hispanique, $\mathrm{n}^{\circ}$ XXVI, Bordeaux, 1924.

3 Tel est, en effet, le sens de son nom hellénique, Herakles. 
indubitablement populaire de l'âge classique en plein rayonnement ?

Hercule ${ }^{4 a}$, c'est pour nous ce qu'il était à Thèbes et en Argos, les deux berceaux de sa légende : la personnification de la force ; mais à mesure que ses courses lui ouvraient de nouveaux territoires, son image s'enrichissait, semble-t-il, de nouveaux attributs, dérivés du premier : pour ce qui est de l'ouest du bassin où s'étend son influence, on le retrouve à Rome, où on le célèbre sous les invocations de Victor - Vainqueur, voire Triomphateur-, de Pacifer-Porteur de Paix, c'est-à-dire Dissuasif -, de Defensor, etc. Il est devenu là protecteur du sol, dieu de l'abondance, et même des trouvailles et de la bonne foi : voici poindre avec une singulière clarté les raisons fondamentales de son patronage du mythique palais tolédan; et elles sont de provenance latine - si encore il ne faut pas remonter plus haut.

Car, dès le III siècle avant notre ère, Rome conquiert ce qui est maintenant l'Espagne, contrée avec laquelle le héros, au témoignage de son épopée primitive, a tissé des liens tout spéciaux : il a vaincu le roi-monstre Géryon, maître de l'île d'Erythie, qu'on situe généralement au large de Gadès, en pays tartessien; il a écarté Calpé et Abyla pour créer les fameuses Colonnes, qui ne sont autres que les rochers de

4 Peu après avoir écrit ce qui précède, nous avons vu notre idée indirectement, mais solidement corroborée, en découvrant que G. Maspéro, dans sa passionnante Histoire Ancienne des Peuples d'Orient, éd. Hachette, Paris, 1912, l'émettait déjà à propos de Melqarth, l'Hercule tyrien : il «avait [...] dessein de conquérir l'Ibérie [...] Il avait soumis l'Afrique, il y avait introduit l'agriculture [c'est donc, là aussi, un dieu du sol, au pouvoir civilisateur] [...], il avait franchi le détroit auquel il donna son nom, fortifié Gadès et vaincu l'Espagne [...] cette épopée d'ensemble [...] résume assez bien les traits principaux de la colonisation phénicienne [...]»

4a Voir, en particulier, Dictionnaire Encyclopédique, 7 vol., s. d., Larousse, Paris (la date la plus récente qui apparaisse dans cet ouvrage est 1907); et Mythologies de la Méditerranée au Gange, ouvrage collectif sous la direction de P. Grimal, Larousse, Paris, 1963.

5 A ce sujet, voir, par exemple, A. Ammann et E. C. Coutant, Histoire Ancienne de l'Orient et de la Grèce, éd. F. Nathan, Paris, 1905, et M. Sauve, Linguistique Générale et Spécificités Castillanes, section XXIV, Les Origines : n. 2, p. 134 ; éd. Ellipses, Paris, 1997. 
Gibraltar et de Ceuta ; il a aidé Atlas - auquel les monts du Maroc doivent leur nom - dans le jardin des propres petitesfilles de ce dernier, les Hespérides : leur père, Hesperos, l'Ouest, a été engendré par lui. Voilà que transparaît une fois de plus ici la réalité proto-historique : ce lieu merveilleux, situé au bord occidental du monde, que serait-ce d'autre que l'Espagne, ou, au comble de l'audace, les Canaries ? Pour l'univers méditerranéen de l'époque, et abstraction faite des explorations phéniciennes vers l'Irlande et peut-être l'Orénoque ${ }^{5}$, c'est bien là, à peu près, que doit se creuser l'abîme du ponant ; au reste, l'une des Hespérides n'est-elle pas Erytheia ? L'Erythie de Géryon, quelquefois assimilée à la péninsule, et l'île des Hespérides pourraient donc fort bien ne faire qu'une. Et, à tant de signes d'un très précoce enracinement ibérique de la figure d'Hercule, ne doit-on pas ajouter cette étrange coïncidence : les combats tauromachiques que connaît l'ère moderne répondent, selon les spécialistes, à une hiératique solaire, tout comme les rituels minoens. Or, l'un des douze travaux imposés au surhomme fut, précisément, de vaincre le taureau crétois - celui de Pasiphaé ou un autre ; animal assez indéterminé pour être emblématique : celui-là, finalement, que, lors des actes cérémoniels, à Cnossos, les jeunes gens devaient, en de savantes culbutes, savoir survoler au péril de leur vie ; les jeux de l'arène apparaissent dès lors comme le vestige le plus concrètement persistant de l'action que la légende attribue à Herakles sur le pays du couchant.

Ainsi, tout exotique qu'elle paraisse à ce moment - ce qui n'est certes pas étranger à sa puissance poétique -, la résurgence d'une tradition herculéenne en terre castillane, dans un contexte où, au XIVe siècle, il s'agit, par tous les moyens, de rendre plausible l'affront subi jadis par ce qui s'avère être l'orgueil national naissant, ne peut, à la réflexion, surprendre : maintenant affublé, du reste, d'un providentiel et fort local neveu, Espán ${ }^{6}$, le Héros par excellence est aussi, par

6 En aucun texte de mythologie gréco-latine à visée universaliste il ne se voit affligé d'une telle parenté : il n'y a d'Hispanus nulle part ; seul existe, et dans l'imagination de l'érudit scripteur de la Chronique, auquel on pourra ajouter, ad libitum, tel ou tel conteur dont il s'inspire, 
droit du sol, celui de cette terre : garant de sa richesse, de sa prospérité, et même de sa bonne foi - de l'honneur de ses habitants - il l'a prise sous son égide, et la maison qu'il y a réalisée, parmi d'autres merveilles que notre texte ne nous fait pas l'injure d'énumérer - car ce sont, par chance, «ces grandes choses que tout le monde connaît $»^{7}-$, est le symbole, en même temps que le saint des saints, des valeurs du royaume, qu'il faut affirmer face à l'Ennemi. Du coup, l'immédiate conséquence de leur violation par Rodrigue est la sarcastique mise en vedette de l'invocation latine d'Hercule en tant que dieu des trouvailles: tardive, mais sans doute bien délectable petite revanche du clan des Romains sur celui des Goths8.

Singulière autant que subite, cependant, l'envie qui frappe le nouveau roi ; car nouveau, il l'est même, au début, au point de ne rien savoir de l'édifice; si l'on a saisi, ce doit pourtant bien être l'un des plus remarquables de sa capitale ; or, à cet instant, sa science n'égale même pas celle du chroniqueur: sa lamentable ignorance rejoint, en fait, la nôtre. Heureusement, les gardiens du temple - au nombre sacré de la complétude et de la totalité, à l'instar des apôtres et des pairs de Charlemagne : douze, comme nous l'apprendrons un peu plus tard - sont là pour parfaire l'éducation du rustre parvenu, et établir la vérité ; car c'est leur témoignage seul qui fonde, chez le roi comme chez le lecteur, cet engagement minimal de foi en la réalité sensible de l'objet que le récit propose, sans lequel il n'y aurait point d'événement : « ceux qui gardaient la maison de Tolède vinrent au roi sire Rodrigue, et lui dirent ceci : 'Seigneur, nous venons à toi, te requérir de faire, toi, ce que firent tous les rois qui avant toi régnèrent en Espagne : convient à toi de mettre ton cadenas sur cette fameuse maison qu'Hercule fit à Tolède [...]' Et le roi leur demanda q... quelle maison était celle-là dont telles choses lui disaient [...] Et eux lui dirent : 'Seigneur, ceci te dirons-nous de fort bonne grâce,

le bon Espán, au nom de prodigieux hasard, et qui doit être bien aise de pouvoir se rendre si utile.

7 «[...] aquellas cosas que todo el mundo sabe [...]».

8 On consultera en particulier sur cette question le très riche ouvrage de E. A. Thompson, Los godos en España, Alianza Editorial, Madrid, 1971. 
car bien savons la vérité là-dessus' " ${ }^{9}$. De fait, où trouver meilleurs informateurs ? Ces gens, au reste peu encombrés par la modestie, assurent tout connaître, des circonstances de la construction à l'aspect extérieur, par lequel commence, non sans quelque logique, la description.

Mais là, très vite, les choses se gâtent : "Hercule, expliquent-ils, [...] fit à Tolède une maison si subtile et de si grand'maîtrise, que nous ne te saurons dire comment elle est faite ni de quel cerveau [elle sort] $\gg 10$.

Tout en maintenant, dans l'élan du discours rapporté, une cohérence linéaire assez acceptable pour que les contradictions ne s'y manifestent d'abord que sous la forme du malaise, qu'elles provoquent, de l'esprit, créant ainsi, grâce au temps de réflexion nécessaire à un retour mental en arrière, un hiatus entre l'instant de réception du message et celui de la prise de conscience claire de son contenu, ces deux lignes suffisent à renverser toutes les données que l'on avait cru acquises. Car ceux qui, il y a si peu, laissaient entendre qu'ils détenaient "là-dessus » un savoir quasi-encyclopédique, doivent à présent admettre une lacune si énorme que leur crédibilité en est, du coup, fort ternie ; d'autant qu'ils affirment dans la même phrase et le rôle d'Hercule en tant qu'artisan-concepteur de la maison, et leur incapacité à dire à quel génie on la doit ; ce qui met en cause non plus tant la qualité de leur information que la vivacité-même de l'intelligence avec laquelle ils ont pu la recueillir, et la transmettre : dès lors, leur rapport est-il bien fiable?

Ce n'est là qu'un procédé coutumier à la narrative médiévale, nous dira-t-on : l'exposition des circonstances n'y est jamais exhaustive d'emblée lorsqu'il s'agit d'évoquer cet

9 Dans la graphie que lui prête Menéndez Pidal, le texte est le suivant : « [...] los que guardavan la casa de Toledo venieron al rey don Rodrigo e dixiéronle así: 'Señor, nos venimos a tí a te requerir que tú fagas lo que fizieron todos los reys que ante ti regñaron en España, que a ti conviene de echar el tu candado en aquella casa que Hércoles fizo en Toledo [...]' E el rey les preguntó que qué casa era aquella de que así le dezían [...] E ellos le dixieron: 'Señor, esto te diremos nos muy de grado, ca bien sabemos dello la verdad [...]' »

10 «[...] Hércoles [...] fizo en Toledo una casa tan sotil e por tan grant maestría que non te sabremos dezir cómmo es fecha nin por cúyo seso $;[\ldots] »$ 
instant de surprise qui coupe le souffle devant une merveille qu'on découvre ; cela permet de revenir, dans un deuxième temps, en étroit accord avec la démarche réelle de l'esprit, à l'analyse plus objective des composants du concret; sans doute ; mais l'emploi de cette technique, que nous verrons, plus loin, beaucoup mieux se justifier, est ici d'une maladresse insigne, puisque les ressorts en sont exactement inversés, l'assurance précédant le trouble sans qu'entre temps, aucune surrection d'élément extérieur ne puisse expliquer une telle évolution; il en résulte que « ceux qui gardaient la maison de Tolède » 11 , si solennellement venus vers l'usurpateur, apparaissent collectivement comme de fats personnages, simplement préoccupés de voler au secours du succès, en proposant au nouveau maître de satisfaire au plus tôt à un rituel de confirmation qu'il ne soupçonnait même pas ; et finalement, du fait de l'initiative qu'ils prennent, d'évidence, trop précipitamment, on ne peut échapper au sentiment qu'ils ont une lourde part de responsabilité dans la catastrophe ultérieure.

Après ce préambule, voici l'image de la maison : elle « est toute ronde, car [...] elle ne [...] paraîtra autre qu'une cuve qui est dressée sur le fond ${ }^{12}$. Personne ne saurait en décrire l'intérieur, mais pour ce qui est de l'extérieur, nos avisés spécialistes se font fort d'en détailler les particularités : «il n'y a pierre qui semble posée de main d'homme, et bien entendons que toutes - la plupart - sont des marbres, et elles sont si éclatantes que c'est merveille, et de tant de couleurs, et telles, qu'à grand'peine pourrez penser qu'elles sont là deux ni trois pierres d'une même couleur, et sont si subtilement jointoyées que, n'étaient les couleurs, bien semblerait, à ceux qui les verraient, que toute la maison fût d'une seule pierre.

11 On remarquera l'emploi, devant casa, en cet instant du texte où l'unité en cause n'est en rien constituée pour le lecteur, de l'article d'universel II de Guillaume, par quoi il est clairement notifié que seul le point de vue du chroniqueur est ici ressenti comme digne de considération. Sur la notion de constitution d'unité, et le système de l'article espagnol en général, on pourra consulter, par exemple, notre Article imperfectif, dans les Actes du Ve Colloque de Linguistique Hispanique d'Aix-en-Provence, publiés en 1994.

12 «[...] es toda redonda, que [...] non [...] paresçerá sinon una cuba que está levantada sobre el témpano $[\ldots] »$ 
Or, les scènes historiées qui transparaissent en elle, cela vous paraîtrait chose fort malaisée à croire, à moins que ne le vissiez; et ne croyez point qu'elles sont peintes à teint, mais les pierres sont ainsi disposées et placées en telle configuration qu'il vous semblera qu'au monde jamais n'y eut bonne chevalerie dont n'y ait là l'histoire. ${ }^{13}$

Edifice de plan circulaire, sans une fenêtre - visible du moins ", fait de petites pierres de marbre éclatant, mais toutes - enfin, la plupart - de couleurs infiniment variées qui, sans pouvoir se mélanger - ainsi que le feraient les nuances du prisme sur une palette pour produire le blanc le plus pur-, ensemble se fondent en une seule : on dirait d'un monolithe monocolore où passent néanmoins, et s'inscrivent, les rêves de toutes les Reines Mathilde. A l'âge où, dans la vérité des jours, le gothique jette au ciel ses flèches et cisèle, sur le moindre chapiteau, une flore luxuriante, tandis qu'aux palais andalous se multiplient les arcs outrepassés, il faut admirer les capacités visionnaires qui se révèlent en ces lignes épurées, où courent, infinis lithogrammes, des bandes dessinées contant, seule concession à l'époque, les exploits de toute franche chevalerie - qu'Hercule, naturellement, premier des preux, pouvait prévoir. Nous sommes là, dans l'esprit, de par la conception de l'art, bien plus près d'un mastaba à Tell-elAmarna que de la cathédrale de Burgos ou de l'Alhambra de Grenade.

Cela étant, on le voit, les contradictions ne manquent pas dans la description du monument. Et c'est son aspect qui est en cause, beaucoup plus que sa structure : une cuve reposant sur le fond, cela tient effectivement debout ; c'est, pratiquement, une tour qui ne dit pas son nom, et évoque, étrangement, celles des hauts échangeurs des centrales atomiques

13 « [...] non ha piedra que semeje que mano de omne la oviese puesto, e bien entendemos que todas las más son mármoles, e son tan claras que es maravilla, e de tantas e tales colores que a duro podredes pensar que aí están dos nin tres piedras de un color, e son así sotilmente ayuntadas que bien semejaría a los que las viesen, si las colores non fuesen, que toda la casa era de una piedra. Mas las estorias que en ella paresçen, esto vos paresçería grave cosa de creer a menos que lo viésedes; e non creades que son pintadas con tinta, mas las piedras son así metidas e puestas de tal figura que vos semejará que nunca en el mundo ovo buena cavallería de que allí non aya la estoria. » 
actuelles. Mais comment, de la réunion de gemmes de multiples teintes, en quel ordre assemblées sur l'immense aire cylindrique - à moins qu'elle ne soit, après tout, conique, puisque ce qui nous est donné à voir, c'est la projection dans l'espace d'un objet de section ronde - naît l'impression d'unicité ? Transposition des conceptions issues de l'enseignement théologique du temps, ou simple effet physique de l'éblouissement ? Et comment, de cette surface si laborieusement ouvrée pour y créer l'uni, peuvent se détacher tant de nobles figures, aux yeux de qui la contemple? On ne nous l'envoie pas dire : il faut le voir pour le croire.

Laissons donc cela : ce n'est point encore l'essentiel, puisque la représentabilité mentale y est, à peu près, assurée. Oublions également les quatre énormes lions de métal qui soutiennent le tout, et même la porte "pas très grande » pratiquée après coup - ainsi procède l'enfant qui dessine -, puis utilisée, puis cadenassée d'or par Hercule lui-même : ils n'offrent pas matière à d'insurmontables interrogations, encore qu'il s'avère là, un peu tard, que le fond de la fameuse «cuve » ne repose pas sur le sol, et que par conséquent, l'entrée interdite se trouve à plusieurs pieds de hauteur, alors que rien, à aucun moment par ailleurs, ne vient préciser comment se résout la difficulté d'accès, qui est pourtant vaincue par Rodrigue : la situation-même de l'huis, donnant, là-haut, sur le vide, le dit mythiquement inaccessible, mais après tout, au plan des réalités technologiques, ne manque ici que la mention d'un tremplin ou d'un escalier, que volontiers on imaginerait assez semblable à ceux des pyramides à degrés aussi familières à diverses civilisations anciennes du MoyenOrient qu'aux Méso-américains, inconnus des clercs de $1344^{13 a}$.

13a Nous devons à notre collègue et amie Marie-Louise PillardLecourtois, ancienne élève de l'Ecole des Chartes, professeur émérite de lettres classiques au lycée Henri-Bergson à Paris, cette intéressante remarque qu'il puisse y avoir ici, en fait, une allusion à une technique de défense relativement courante au Moyen-Age : elle consistait en ce que, dans certaines tours, l'entrée, située en l'air, nécessitait l'usage d'une échelle, dont disposaient les occupants, et qu'ils retiraient après le passage de ceux qu'ils avaient autorisés à les rejoindre. Deux arguments nous ont paru s'opposer à une telle interprétation; le premier est d'ordre esthétique : il est difficile d'imaginer qu'un objet si 
Quoi qu'il en soit, il ne faut rien d'autre à l'impétueux novice que les clefs des cadenas pour pénétrer dans l'enceinte protégée ; encore, dès qu'il en prend possession, se retrouvent-elles frappées d'inutilité, puisque, sans doute mû par l'impatience «le scripteur ne s'en explique pas clairement - il fait enfoncer la porte, soudain multipliée, du reste, à l'instar, peut-être, de celles qui se succèdent aux tambours des églises $^{14}$ : tant il est vrai qu'il n'est sanctuaire si étroitement clos qu'il puisse résister à quelques solides épaules aux ordres d'un chef entreprenant, à qui le sort, c'est chose depuis longtemps entendue, ne peut qu'être propice: "audaces Fortuna juvat».

Le profanateur n'est pas foudroyé dans son acte. C'est même à travers ses yeux, et ceux des dignitaires de sa suite, que nous allons enfin connaître le grand secret : désormais, tous les points de vue coïncident et se confondent en un seul, neutre, sans spécification, celui du narrateur omniscient ; car c'est le destin des secrets qu'une fois violés, ils deviennent chose publique ; et le vulgaire n'est pas moins digne de les recevoir qu'un roi félon.

Cependant, première surprise, ce qui, de l'extérieur, se donnait pour circulaire, s'avère, à l'intérieur, carré : « Et la maison qui de dehors paraissait ronde, ils [y] trouvèrent un palais [disposé] en carré15»; cela signifie-t-il que notre

rustique vienne s'adjoindre à un tel bâtiment, pétri de tant de fantasmes; le second est pratique; il n'y a personne à l'intérieur, qui puisse remonter les degrés amovibles. Toutefois, rien n'est impossible ; ce ne seraient là, après tout, que deux incohérences de plus, et notre conteur n'est certes pas homme à s'arrêter devant de telles broutilles.

14 Soulignons graphiquement les marques de cette pluralité inopinée: "E él mandó que traxiesen las llaves de los candados, e commo venieron, sin ninguna detenençia, fué a las puertas de la casa e fízolas quebrar..."

15 «E la casa que de fuera paresçía redonda, fallaron un palaçio en quadra "; " un palaçio en quadra » : peut-être faut-il comprendre " un palais disposé comme une salle carrée »: souvent, en langue médiévale, et en tout cas systématiquement dans le Poème du Cid (voir à ce sujet J. Corominas, Diccionario Crítico Etimológico Castellano e Hispánico, ed. Gredos, Madrid, 1980, et R. Menéndez-Pidal, Cantar de Mio Cid, Texto, Gramática y Vocabulario, Espasa-Calpe, Madrid, 1954), quadra a le sens de salle, ce qui implique l'assimilation de toute 
chroniqueur compte, parmi les exploits d'Hercule, celui d'avoir trouvé l'introuvable, la quadrature du cercle ? C'est bien possible, mais non certain ; car il suffit, pour qu'un carré s'inscrive parfaitement dans une circonférence, qu'il n'en occupe pas toute l'aire, et que ses angles y forment quatre points de tangence interne : nous ne sommes point encore au cœur du problème ; mais notre auteur, alors, d'ajouter : « (en carré), tant d'une part que de l'autre ${ }^{16} »$ : les témoins sont éblouis, certes, et ne savent plus où donner des yeux ; toutefois, le premier choc passé, que reste-t-il ?

Le carré s'est-il dédoublé, offrant sa propre image à droite et à gauche des visiteurs ? S'est-il même étendu, ou multiplié, tout à coup, de telle façon que, se trouvant encore à l'entrée de la pièce, Rodrigue et ses proches se sentent, irrésistiblement, absorbés, englobés par l'univers, soudain, où ils ont pénétré ? Tel paraît bien être le cas : après avoir renouvelé l'inévitable aveu de son impuissance à décrire ce que, malgré tout, il a l'ambition de montrer : « [palais] si merveilleux qu'il n'y a homme au monde qui le pût dire ${ }^{17}$, le chroniqueur

pièce carrée, ou au moins quadrangulaire, à un lieu de réunion, domestique ou palatin. Néanmoins, ce serait accepter ici ce que, plus bas, nous rejetons.

16 « ([...] en quadra), tanto de una parte commo de otra, »

17 « tan maravilloso que non ha omne en el mundo que lo [...] podiese dezir"; il s'agit moins, du reste, d'une réelle contradiction que d'une précaution oratoire prise pour couvrir l'imperfection des paroles, et leur inaptitude à rendre compte de la réalité vécue dans sa force : figure qui admet des variantes verbales, mais apparaît avec une irritante fréquence, et non point seulement sous des plumes médiévales ; la tradition castillane en fait grand usage jusqu'à l'âge classique, et Cervantes soi-même, lorsqu'il s'agirait de faire percevoir leur beauté ou leur charme, y trouve souvent un excellent moyen de ne pas décrire les femmes dont il parle.

18 « ca una de las quadras del palaçio era así blanca, que la nieve non lo podría más ser; e la otra quadra del palaçio que era en derecho della era tan negra [...] que más non podiese ser ; e la otra parte era tan verde commo una muy verde esmeralda [...] e la otra parte del palaçio, que era en contra desta, era tan clara commo si fuese un fino cristal $[\ldots] »$

19 «E así commo de la parte de fondón era [el palaçio] muy bien llano, así vieron que de la parte de ençima era muy plano, mas que avía y finiestras $[\ldots]$ »: "Et tout comme du côté du sol - interprétation 
explique : «car l'un des carrés du palais était d'un tel blanc, que la neige ne le pourrait davantage être ; et l'autre carré du palais qui était face à lui était si noir [...] que davantage n'eût pu être ; et l'autre partie était aussi verte qu'une fort verte émeraude [...] et l'autre partie du palais, qui était à l'opposé de celle-ci, était aussi éclatante que si elle eût été de fin cristal ${ }^{18}$.

Point d'orgue ; la suite du texte fait bien état de fenêtres haut placées, de telle sorte qu'elles forment peut-être même verrière en terrasse, ce qui préfigurerait certaines des plus modernes techniques de l'architecture actuelle, et expliquerait pourquoi ces ouvertures sont invisibles de l'extérieur - on ne sait trop quelles images, au juste, faire correspondre aux propos du scripteur, sur ce point redoutablement extensifs ${ }^{19}-$, mais peu importe ; car c'est ce " palais carré » qui, de par sa division subreptice en quatre carrés dépendants, pose, sous des dehors, en somme, bénins, l'énigme fondamentale, celle qui concerne la représentation de l'espace. Est-ce là de grand art, ou bien faut-il encore une fois mettre l'accent sur une maladresse? Toujours est-il que, tel quel, le texte nous transporte dans l'incontrôlable absolu, grâce à son absurditémême : il projette personnages et lecteurs - dès lors pris aux rets de l'assimilation sensitive - dans une situation brute, qui s'impose, inexpliquée, inexplicable, de par la puissance de sa propre existence ; donnée immédiate convoquée par l'esprit du prodige, et vécue pleinement du simple fait de son évocation, mais où ne subsistent que des bribes de logique.

Ecartons tout d'abord l'hypothèse d'où dériveraient les difficultés les plus grandes, même si c'est sans doute la mieux défendable sur le plan linguistique, et refusons de voir, sous le mot quadras, des salles à part entière ; car une salle a des murs ; dès lors, d'où et comment les intrus pourraient-ils embrasser d'un seul regard les quatre parties ressenties, sinon décrites, comme simultanément découvertes ? Même l'aberra-

qu'autorise P. de Corral, puisque, dans le même contexte, il emploie "suelo" - [le palais] était fort remarquablement plat, ainsi virent-ils que du côté du haut, il était très plan, mais qu'il y avait là des fenêtres ». 
tion onirique la plus profonde paraît devoir refuser de tels obstacles.

Le carré originel s'est donc subdivisé à vue, deux fois, comme par magie, comme une entité douée de vie : ainsi, la cellule, ayant atteint la taille adéquate, donne naissance à deux cellules égales. Tels les quatre cantons d'un blason, quatre carrés occupent maintenant Rodrigue, l'entourent peut-être - où est-il exactement par rapport à eux ? Chacun est de couleur différente - quelle était celle du carré d'origine ? Les couleurs sont opposées deux à deux - mais pourquoi au noir et au vert le blanc et le cristal, deux modalités de l'incolore?

Le noir du malheur et du deuil annoncés équilibrant le blanc de la jeunesse et de l'innocence, cela se comprend, à condition de voir, sous l'immaculé, l'image de l'Espagne, et non celle de son roi, qui est coupable. Mais que penser de ce vert, symbole admis de l'espérance et du renouveau, appelé à faire pièce au cristal, qui, pour sa part, ne dit que la transparence, la sincérité, et peut-être la fragilité ? Le combat suggéré par le premier couple, qui pourtant dit une intention structurelle bien claire, n'est pas ici prolongé dans le second : il faut chercher plus avant.

En fait, aux deux symboles de limpidité, qui disent l'Espagne, sont affrontés ceux de l'Islam: car au vert canonique, s'ajoute le noir - souvenir lointain de la pierre de la Ka'ba et du pélerinage à La Mecque, ou bien, plus proche, des bannières du Lamtuna Yusuf al Teshufin, et de la terreur surgie du grand sud saharien avec cette irrépressible méharée rigoriste qui avait si bien failli submerger la totalité des territoires chrétiens ? En 1344, il y avait de cela à peine trois siècles : temps court, pour une civilisation de tradition essentiellement orale... 
Tout est donc dit, avant même qu'ayant repris leurs esprits ${ }^{20}$, les témoins n'avisent, enfin, au beau milieu du palais, l'arche en forme de tronc de colonne ${ }^{21}$ où est déposé le secret des secrets, qui scellera le destin du roi. Tout ? Pas encore, à vrai dire, car une question demeure: face aux couleurs islamiques - celle du Coran et celle des corsaires de sa cause, l'Espagne est, symboliquement, plus qu'innocente: déjà exsangue, libérée de tout mal ; comme si la faute de son bouillant chef était déjà dépassée, absorbée, perceptible seulement en des conséquences plus que tardives. L'affrontement est donc, pour ainsi dire, déséquilibré, parce qu'évoqué en une singulière a-synchronie : comme si, dans la perspective herculéenne, la percée de l'Islam avait appartenu à un futur simple, de désastre - rendu présent, ainsi que dans toute prophétie, par sa propre force -, à partir de quoi se fût défini, dans l'autre camp, un futur ultérieur, apaisé, celui de la sainteté retrouvée à travers l'épreuve, celui de la pureté nécessaire à de nouveaux épanouissements ${ }^{22}$.

En fin matois, naïf à ses heures, syncrétique plus que logique, et doté d'un incontestable sens du merveilleux, notre scripteur, à travers un univers parfois un peu bancal, sait parfaitement nous mener où il veut que nous allions ; c'est au reste le grand charme de son art : voici apparaître, au détour d'une énigme posée là comme on tend un piège, telle

20 «tovieron mientes", « ils reprirent leurs esprits », écrit exactement le chroniqueur, décidément très attentif à traduire les mouvements de l'âme, pour ne pas dire les réactions psychologiques, des visiteurs devant tout ce qui s'offre à leurs yeux ébahis.

21 Le texte indique : "vieron estar un estelo non muy grueso e era todo redondo, e era tan alto commo un omne, e avía en él una puerta muy sotilmente fecha e asaz pequeña "; bien qu'estelo ne semble guère avoir attiré l'attention des lexicographes, il faut, de toute évidence, ici, rapprocher ce mot de estela, en son acception de monument commémoratif, qui peut affecter la forme de cippe ; néanmoins, particularité très remarquable, l'objet est conçu par le scripteur comme creux, au moins en partie, et muni d'une " porte [...] fort petite ", ce qui en fait une sorte de tabernacle.

22 Plus sensible, sans doute, à l'illogisme chromatique, ou à l'anachronie, de cette situation qu'à sa signification profonde, P. de Corral substitue, pour sa part, le rouge de la passion, du sang et de la destruction au prometteur cristal de la Chronique. 
maladresse verbale, soudain, finement calculée en fonction de la conclusion à suggérer - à illustrer, peut-être, grâce à des images - sans toutefois l'exprimer. Car ce texte, comme presque tous ceux, sans doute, que le Moyen-Age a produits, a fonction utilitaire : politique ; encore l'effort de le saisir est-il, afin qu'il atteigne mieux son but, exigé de son lecteur - ou de ses auditeurs. 